\title{
A Combining Forecasting Modeling and Its Application
}

\author{
Dengmei Qiu and WenJie Wang* \\ Glorious Sun School of Business and Management, Donghua University \\ Shanghai, 200051, China \\ \{dmqiu, wenjiew\}@dhu.edu.cn
}

\begin{abstract}
The supply chain coordination has abstracted more and more attention from industries and academics. This paper studies a Bayesian combination forecasting model to integrate multiple forecasting resources and coordinate forecasting process among partners in retail supply chain. The simulation results based on the retail sales data show the effectiveness of this Bayesian combination forecasting model to coordinate the collaborative forecasting process. This Bayesian combination forecasting model can improve demand forecasting accuracy of supply chain.
\end{abstract}

Keywords: Combination forecasting, Bayesian model, Supply chain.

\section{Introduction}

With the development of information and network technology, various innovative supply chain solutions are created. Collaborative planning, forecasting and replenishment (CPFR), which is an retailing supply chain coordination innovation based on the network technology, has been adopted and implemented by many world-renowned retailers and manufacturers, such as Wal-Mart, Proctor \& Gamble, etc.. CPFR concerns the collaboration where two or more parties in the supply chain jointly plan a number of promotional activities and work out synchronized forecasts, on the basis of which the production and replenishment processes are determined [1]. The first CPFR project was piloted by Wal-Mart with its suppliers in 1995. The results of two-year project showed that CPFR could simultaneously reduce inventory levels and increase sales for both retailers and suppliers. Since its original application was initiated, CPFR has had many successful applications in North America, Europe and China [2].

The collaborative forecasting plays an important part in CPFR implementation procedure. We will briefly review the CPFR concept and its implementation process at the beginning of this paper. And then, the collaborative forecasting process which is the core part of CPFR will be mainly discussed. As the basics phase of the implementation of CPFR, the collaborative forecasting process is the cornerstone to the success of

\footnotetext{
* Corresponding author.
} 
CPFR projects. The collaborative forecasting process of CPFR requires a solid forecasting approach to synthesis information and knowledge from multiply parties in the supply chain. The combination forecasting method can combines forecasting models from different parties to smooth coordination in the supply chain and reduce forecasting discrepancies. Thus, considering the multiple forms of forecasting resources in the retail supply chain, the Bayesian combination forecasting method is applied for CPFR collaborative forecasting modeling with improved forecasting accuracy and supply chain collaboration performance in this paper.

Combining forecasts is a well-established procedure for improving forecasting accuracy which takes advantage of the availability of both multiple information and computing resources for data intensive forecasting [3]. Since Bates-Granger first proposed the combination forecasting method in 1969 [4], many kinds of combining methods have been developed [3]. Zeng et al [5] studied the combination forecasting model with error correction and changing weight coefficient. Hoogerheide et al [6] compared several Bayesian combination schemes in terms of forecast accuracy and economic gains. Bayesian combination methods use the distributional properties of the individual forecasts to construct the combination. The demand forecasting in retail supply chain is impacted by many factors such as product promotion or social development trend. And, subjective forecasting based on the expert experiments is often used in retail market forecasting. So, the Bayesian combination forecasting model is considered as the collaborative forecasting approach in retail supply chain coordination.

In the first part of this paper, the CPFR retail supply chain coordination and collaborative forecasting process are discussed briefly. In the second part of the paper, a Bayesian combination forecasting method is modeled to coordinate forecasting process in retail supply chain. Finally, the simulation for this model is completed based on the Carrefour sales data. The simulation results showed the effective of this Bayesian combination forecasting model in retail supply chain collaboration process.

\section{Bayesian Combination Forecasting Modeling for Collaborative Forecasting Process}

The combination method proposed by Bates and Branger in 1969 is normally called as optimal linear combination model or B-G method. The forecasting results $f_{c 1}, f_{c 2} \cdots f_{c n}$ are supposed as random variables with the covariance matrix $\sum$ in this model. Based on the minimizing the variance criteria (MV), the optimal forecasting results can be calculated as the following formula (1)

$$
f_{c}=\sum_{k=1}^{n} W_{k} f_{k}=W^{T} \bullet f
$$

Here, weighting vector $W^{T}=\left(1_{n}^{T} \sum^{-1} 1_{n}\right)^{-1} 1_{n}^{T} \sum^{-1}=\left(W_{1}, W_{2} \ldots, W_{n}\right), \quad 1_{n}^{T}=(1,1, \ldots, 1)$.

The Bayesian combination forecasting model is developed based on the B-G method and uses the distributional properties of the individual forecasts to construct the 
combination. Supposed the $\mathrm{Y}$ is presenting the samples of actual demand. The forecasting results obtained from the different parties in the supply chain which are forecasted with $m$ kinds of individual forecasting methods are presented by $f_{1}, f_{2}, \cdots f_{m}(j=1,2, \ldots, m) . \quad i=1,2, \cdots, n$ present different forecasting time periods. The Bayesian combination forecasting method makes use of the Bayesian rule to decide the optimal combination ways and weights of individual forecasting methods in combination model and get the combined forecasting results in different time periods $\hat{f}_{i c}(i=1,2, \cdots, n)$, which can be fully approximated to actual demand values.

Set $Z_{i}^{T}=\left(f_{1}, f_{2}, \cdots f_{m}\right), i=1,2, \cdots, n$, and then the joint probability density function of $\mathrm{m}$ individual forecasting samples on independent time $z_{1}, z_{2}, \cdots, z_{n}$ can be calculated as follows.

$$
f\left(z_{1}, z_{2}, \cdots, z_{n} \mid Y ; \varphi\right)=\prod_{i=1}^{n} f\left(z_{i} \mid Y_{I} ; \varphi\right)
$$

Here $\varphi$ is parameter vector, and $Y^{T}=\left(y_{1}, y_{2}, \cdots, y_{n}\right)$ is the vector of the actual demand samples on the different forecasting time periods.

According to the Bayesian rule, the probability density function that $\mathrm{Y}$ is specified as a vector value $y$ is as follows:

$$
\begin{aligned}
& f\left(y \mid z_{1}, z_{2}, \cdots, z_{n}\right)=\frac{\prod_{i=1}^{n} f\left(z_{i} \mid y_{i}\right) f\left(y_{i}\right)}{\prod_{i=1}^{n} \int_{A_{I}} f\left(z_{i} \mid y_{i}\right) f\left(y_{i}\right) d y_{i}} \\
&=\prod_{i=1}^{n} \frac{f\left(f_{i 1}, f_{i 2}, \cdots f_{i m} \mid y_{i}\right) f\left(y_{i}\right)}{\int_{A_{i}} f\left(f_{i 1}, f_{i 2}, \cdots f_{i m} \mid y_{i}\right) f\left(y_{i}\right) d y_{i}}
\end{aligned}
$$

Here $f\left(y_{i}\right)(i=1,2, \cdots, n)$ is the prior probability distribution of $y_{i}$, which presents the prior estimation or preference of decision maker to $y_{i}$. $A_{i}$ is the definition set of $y_{i}$.

If only one forecasting time period is considered as the general case and prior distribution $f(y)$ is uniform distribution form, that is $f(y) \propto 1$, the formula (2) can be simplified as the following.

$$
f\left(y \mid f_{1}, f_{2}, \cdots, f_{m}\right)=\frac{f\left(f_{1}, f_{2}, \cdots f_{m} \mid y\right)}{\int_{A} f\left(f_{1}, f_{2}, \cdots f_{m} \mid y\right) d y}
$$

The forecasting error distribution of individual forecasting results $\left(f_{1}, f_{2}, \cdots f_{m}\right)$ is chosen as normal distribution or logarithm normal distribution in most case. In this paper, more general distribution of forecasting error is introduced through Box-Cox conversion. 


$$
Z^{(\lambda)}=\left\{\begin{array}{cc}
\frac{Z^{\lambda}-1}{\lambda} & \lambda \neq 0 \\
\ln Z & \lambda=0
\end{array}\right.
$$

Here, $\lambda$ is conversion parameter

Supposed $\sum$ is the covariance matrix of $\left(f_{1}, f_{2}, \cdots f_{m}\right)$ and Wi presents the weights of individual forecasting $f i$. Then, weights can be calculated as following formula based on the minimum error variance criteria:

$$
W^{T}=\left(1_{m}^{T} \sum^{-1} 1_{m}\right)^{-1} 1_{m}^{T} \sum^{-1}=\left(W_{1}, W_{2} \ldots, W_{m}\right)
$$

And, nonlinear combination forecasting formula can be obtained through Bayesian analysis as follows, which will be used to calculate the optimal combination forecasting results approximated to the actual values $Y$.

$$
\hat{f}_{c}=\left\{\begin{array}{cc}
\prod_{i=1}^{m} f_{i}^{w_{i}} \times e^{\frac{s^{2}}{2}} & \lambda=0 \\
{\left[1+\sum_{i=1}^{m} \lambda W_{i} f_{i}^{(\lambda)}\right]^{\frac{1}{\lambda}}=\left[\sum_{i=1}^{m} W_{i} f_{i}^{\lambda}\right]^{\frac{1}{\lambda}}} & \lambda \neq 0
\end{array}\right.
$$

Here, $\frac{1}{S^{2}}=1_{m}^{T} \sum^{-1} 1_{m}=\frac{1}{\left|\sum\right|} 1_{m}^{T} \sum^{*} 1_{m}$, and $\sum_{i=1}^{n} W_{i t}=1$ formula.

And, the optimal conversion parameter $\lambda^{*}$ can be calculated as the following

$$
\lambda^{*}=\arg \min _{\lambda} \sum_{j=1}^{t}\left[Y_{j}-\left(\sum_{i=1}^{n} W_{i} f_{i j}^{\lambda}\right)^{\frac{1}{\lambda}}\right]^{2}
$$

Here, $f_{i j}$ present the forecasting result on $j$ time period by $i$ individual forecasting methods. The weights $W_{i}$ can be calculated by formula (4). The covariance matrix $\sum$ can be determined by prior value or estimated by past proximate samples values.

\section{Simulation}

The simulation of the Bayesian combination forecasting model will be based on the sales data of one kind of biscuit product in Carrefour China in this paper. The detailed sales data of this biscuit product in 39 weeks in Carrefour China is showed in table 1. During the simulation process, the sales data from week 1 to week 28 are used to 
estimate parameters in Bayesian combination forecasting model creation. The sales data from week 29 to week 39 are used to compare with the forecasting results obtained from combination forecasting methods.

Table 1. The Sales Data of Biscuit in Carrefour Supermarket

\begin{tabular}{cccccccccccccc}
\hline Week & 1 & 2 & 3 & 4 & 5 & 6 & 7 & 8 & 9 & 10 & 11 & 12 & 13 \\
\hline Demand & 64 & 78 & 62 & 38 & 59 & 13 & 99 & 82 & 105 & 56 & 56 & 88 & 93 \\
\hline Week & 14 & 15 & 16 & 17 & 18 & 19 & 20 & 21 & 22 & 23 & 24 & 25 & 26 \\
\hline Demand & 100 & 110 & 135 & 124 & 105 & 86 & 117 & 172 & 185 & 169 & 192 & 195 & 156 \\
\hline Week & 27 & 28 & 29 & 30 & 31 & 32 & 33 & 34 & 35 & 36 & 37 & 38 & 39 \\
\hline Demand & 136 & 132 & 183 & 84 & 92 & 119 & 137 & 107 & 80 & 218 & 167 & 156 & 170 \\
\hline
\end{tabular}

Based on the Carrefour biscuit sale data and statics analysis, the Bayesian forecasting model can be created following three main steps, which includes proper individual forecasting methods selection, combination ways determination and optimal parameter estimation. The characteristics of the individual forecasts combining in the model has substantial implications on the overall forecasting performance of model, and thus it is very important to make a rigorous analysis on the individual forecast errors. The first step of combination modeling is to compare and select proper individual forecasting methods for combination.

Table 2. Forecasting Results of Five Individual Forecasting Methods

\begin{tabular}{ccccccc}
\hline Week & $\begin{array}{c}\text { Actual } \\
\text { Demand }\end{array}$ & F1 & F2 & F3 & F4 & F5 \\
\hline 29 & 183 & 141.3333 & 138.9865 & 179.2469 & 136.3747 & 125.3344 \\
30 & 84 & 150.3333 & 165.3946 & 184.8024 & 173.2965 & 159.2878 \\
31 & 92 & 133 & 116.5578 & 190.4484 & 112.6916 & 68.3652 \\
32 & 119 & 119.6667 & 101.8231 & 196.185 & 93.30308 & 111.0044 \\
33 & 137 & 98.3333 & 112.1293 & 202.0121 & 115.4397 & 106.5959 \\
34 & 107 & 116 & 127.0517 & 207.9297 & 135.7434 & 117.6981 \\
35 & 80 & 121 & 115.0207 & 213.9379 & 118.0299 & 93.1682 \\
36 & 218 & 108 & 94.0083 & 220.0367 & 90.26195 & 82.5842 \\
37 & 167 & 135 & 168.4033 & 226.2259 & 186.0273 & 177.1399 \\
38 & 156 & 155 & 167.5613 & 232.5058 & 183.4052 & 115.0543 \\
39 & 170 & 180.3333 & 160.6245 & 238.8761 & 162.1665 & 150.9677 \\
\hline
\end{tabular}

In general, different parties in the retail supply chain may use the different patterns of individual forecasting. So, the different patterns of individual forecasting methods, which include the simple moving average, the exponential smoothing, the trend extrapolation method, ARIMA (autoregressive integrated moving average) method and artificial neural network method, are applied to combine model to forecast Carrefour 
biscuit demand from week 29 to week 39. Through comparison study of forecasting results of each individual forecasting method, the best parameters of each individual forecasting method are estimated. The forecasting results of five different individual forecasting methods are indicated in Table 2. The F1 row data indicated the best result forecasted by the simple moving average method when moving period $\mathrm{N}$ equal to 3 . The F2 row data indicated the best result forecasted by the exponential smoothing method when smoothing coefficient $a$ equal to 0.6 . The F3 row data indicated the best result forecasted by the two polynomial regression method $(n=2)$. The F4 row data indicated the best result forecasted by the ARIMA method when parameter d equal to 1 . The F5 row data indicated the best result forecasted by the artificial neural network method when there are three neurons and two hidden layers in the neural network.

After the five individual forecasting methods selected, the second step of combination modeling is to determine proper combination ways of these individual methods. With the Matlab simulation tool, the forecasting results are calculated using different combination ways of these individual forecasting methods. As it is widely accepted that only "good" forecasts should be included in a combination, strong differences in forecast error variances between the individual forecasts are not to be expected [7]. Four individual methods (F1、F2、F4、F5) which have good forecasting performance is decided to be combined into Bayesian combination model.

The optimal conversion parameter $\lambda^{*}$ can be calculated according to the formula (8). The sum of square of forecasting error is minimized when $\lambda^{*}=6.9419$. The optimal conversion parameter $\lambda^{*}$ could be simplified as $\lambda^{*}=7$ in the Bayesian combination modeling. The forecasting error between forecasting result and actual demand are used as the evaluation standards of forecasting methods performance. There are many kinds of measure indexes of forecasting errors [8]. In this paper, four main measure indexes of forecasting error, which are the squares sum error (SSE), the mean square error (MSE), the mean absolute percentage error (MAPE) and the mean square percentage error (MSPE), are applied to comprehensively evaluate the forecasting accuracy of Bayesian combination forecasting model.

Table 3. The Comparison of Bayesian Combination Models Forecasting Accuracy

\begin{tabular}{ccccc}
\hline Combination $\quad$ Forecasting & SSE & MSE & MAPE & MSPE \\
\hline Simple Average Method & $2.64 \mathrm{E}+04$ & $2.40 \mathrm{E}+03$ & 0.3589 & 0.3725 \\
Optimal Linear Method & $2.52 \mathrm{E}+04$ & $2.29 \mathrm{E}+03$ & 0.2734 & 0.3416 \\
Bayesian Combination $\lambda^{*}=7$ & $1.92 \mathrm{E}+03$ & $1.74 \mathrm{E}+02$ & 0.0803 & 0.0561 \\
\hline
\end{tabular}

The Bayesian combination models with different parameters $\lambda$ are compared with the simple average method and optimal linear methods in the simulation based on Carrefour biscuit sale data. The forecasting errors of different combination forecasting methods are showed in the table 3. It can be found that the four measure indexes of forecasting error of optimal Bayesian combination method when $\lambda^{*}=7$ are lower than those of simple combination methods. So, the optimal Bayesian combination forecasting model performs better than other combination models in retail collaborative 
forecasting process. This simulation research proved that the optimal Bayesian combination forecasting method is an effective approach to integrate and coordinate the forecasting process among partners in retail supply chain. Bayesian combination forecasting method can highly improve the demand forecasting accuracy of collaborative forecasting activity in the retail supply chain.

\section{Conclusion}

The collaborative planning, forecasting and replenishment framework provides the practical roadmap for retail supply chain coordination. The collaborative forecasting is taken as the core part in CPFR solution implementation. A Bayesian combination forecasting method, which can combine individual forecasting methods from different parties in the retail supply chain, is modeled for CPFR collaborative forecasting process. The simulation results showed that forecasting discrepancies are reduced and collaborative forecasting accuracy is improved after integrating forecasting process with the optimal Bayesian combination forecasting model. It is turned out that the Bayesian combination forecasting method is an effective means for collaborative forecasting process in retail supply chain. The further research on collaborative forecasting methodology for supply chain coordination will be extended into different statistic features of product demand situation in the future.

Acknowledgments. This research was supported by a grant from the Shanghai Science Foundation Council (12ZR1400900) and the Chinese National Science Foundation Council (71172174).

\section{References}

1. Liu, X., Sun, Y.: Information Integration of CPFR in Inbound Logistics of Automotive Manufactures Based on Internet of Things. Journal of Computers 7(2), 349-355 (2012)

2. Danese, P.: Designing CPFR collaborations: insights from seven case studies. International Journal of Operations \& Production Management 27(2), 181-204 (2007)

3. Bunn, D.W.: Forecasting with more than one model. Journal of Forecasting 8(3), 161-166 (1989)

4. Bates, J.M., Greanger, C.W.J.: Combination of Forecasts. Operational Research Quarterly 20(4), 451-468 (1969)

5. Zeng, Y., Tang, X., Zheng, W.: Combination forecasting based on Stein-rule estimation and error correction. Journal of Management Sciences in China 4(6), 39-47 (2001)

6. Hoogerheide, L., Kleijn, R., Ravazzolo, F., Van Dijk, H.K., Verbeek, M.: Forecast Accuracy and Economic Gains from Bayesian Model Averaging Using Time-Varying Weights. Journal of Forecasting 29(1-2), 251-269 (2010)

7. Menezes, L.M., de, B.D.W., Taylor, J.W.: Review of guidelines for the use of combined forecasts. European Journal of Operational Research 120(1), 190-204 (2000)

8. Zhang, C., Huang, L., Zhao, Z.: Research on combination forecast of port cargo throughput based on time series and causality analysis. Journal of Industrial Engineering and Management 6(1), 124-134 (2013) 